\title{
A Novel Strategy for Complete and Phase Robust Synchronizations of Chaotic Nonlinear Systems
}

\author{
Emad E. Mahmoud ${ }^{1,2, *, t, \neq(\mathbb{D}, \text { M. Higazy }}{ }^{1,3, \ddagger}$ and Ohood A. Althagafi ${ }^{4}$ \\ 1 Department of Mathematics and Statistics, College of Science, Taif University, P.O. Box 11099, \\ Taif 21944, Saudi Arabia; m.higazy@tu.edu.sa \\ 2 Department of Mathematics, Faculty of Science, Sohag University, Sohag 82524, Egypt \\ 3 Department of Physics and Engineering Mathematics, Faculty of Electronic Engineering, \\ Menoufia University, Menouf 32952, Egypt \\ 4 Department of Mathematics, Turabah College, Taif University, P.O. Box 11099, Turabah 22178, Saudi Arabia; \\ a.ahood@tu.edu.sa \\ * Correspondence: e.mahmoud@tu.edu.sa \\ + Current address: Affiliation 1. \\ $\ddagger$ These authors contributed equally to this work.
}

Received: 28 September 2020; Accepted: 21 October 2020; Published: 24 October 2020

check for updates

\begin{abstract}
Our work here is to propose a novel technique by which chaos complete and phase synchronizations can be accomplished via a low-cost scheme. We call the proposed technique a "single-state feedback track synchronization control algorithm". A single-state feedback track synchronization control algorithm is designed so that both complete and phase synchronizations can be accomplished using the same controller. Complete synchronization between two chaotic systems means complete symmetry between them, but phase synchronization means complete symmetry with a phase shift. In addition, the proposed method is applied to the synchronization of two identical chaotic Lorenz models. The effectiveness and robustness of the proposed algorithm is well illustrated via exhaustive numerical simulation experiments based on the Matlab toolbox of the powerful genetic algorithm. The robustness of the proposed algorithm motivated us to apply this method of synchronization in a secure communication application.
\end{abstract}

Keywords: complete synchronization; phase synchronization; track control; genetic algorithm; secure communication

\section{Introduction}

There are two types of chaotic synchronization [1,2]: chaos phase synchronization (CPS) and chaos complete (or full) synchronization (CS). CPS [3-5] is a kind of synchronization by which a chaotic oscillator tunes its internal dynamics' frequencies according to the effects of an external force or to the behaviors of another selected chaotic system, however, the variations of their amplitudes are still uncorrelated. There is a wide range of distinct systems that demonstrate this type of synchronization in physics, chemistry and biology, in addition to coupled electronic oscillating circuits [3], plasma evacuation tubes driven by wave generators with low amplitude [6], globally paired electrochemical oscillator arrays [7], human visual responses that are phase-locked [8] and the flow of blood to adjacent operational units inside the kidney [9].

Considering simple topology systems (e.g., systems whose attractors are of a spiral type and the Rössler system), the chaotic oscillation's phase $\phi(t)$ is defined, in the polar coordinate framework of a suitable projection level $(x, y)$, as an angle where $\phi(t)=\tan ^{-1}\left(\frac{y}{x}\right)$. Generally, the boundedness of the phase difference between two chaotic models implies chaotic phase synchronization between them [10], 
while CS is achieved when the phase difference approaches zero as time approaches infinity. Similarly, the amplitude of the chaotic signal can be defined instantaneously as $A=\left(x^{2}+y^{2}\right)^{\frac{1}{2}}$. Two chaotic models realize CPS in situations where those amplitudes are linearly independent (uncorrelated) and chaotic. Applying Hilbert transformation is sometimes carried out to obtain a more comprehensive definition of the chaotic signals' phase [11]. In addition, the phase may be defined as a fraction of a complete period.

Via numerical trials, we can monitor the method by which the frequency spectrum harmonics for an obliged chaotic system approach the controlling frequency in a selected period. The switch from phase synchronization behavior to non-synchronization behavior of a controlled chaotic system is shown via certain changes in the Lyapunov exponent and via certain variations in the shape of the Poincare map. In theory, the major issue is understanding the regulation of the huge group of unsteady cyclic paths that model the state of chaotic synchronization [12].

The complete synchronization (CS) notion came about through noting [13] that a couple of similar chaotic systems with suitable conditions can synchronize their changes completely, and the status of synchronization can be stabilized under accidental disturbances. This observation has attracted great attention via its possible utilization in safe communication applications that have become very important $[14,15]$. Theoretically, the great attention on CS is due to the several new ideas and phenomenons it has caused, such as soft attraction [16], puzzled and mixed basins of attraction [17], discontinuous intermittence [18] and bubbling attractors [19]. Specific attention is given to the design of the attraction basin for the state of chaotic synchronization [20] and to establishing necessary and sufficient synchronization conditions [21]. The traditional synchronization condition relying on Lyapunov conditions is not necessary, however it is sufficient. In addition, there is an insufficient alternate case that requires the largest transversal Lyapunov exponent to be negative.

From all of the chaotic systems, the chaotic Lorenz model is commonly selected because it involves numerous characteristics of chaotic behaviors [22,23]. For a real framework, it commonly operates in the required stable work regions. Consequently, the tracking of controlled chaotic models (where states are driven to track certain predetermined reference values) has attracted great interest. In [24], a tracking control method was applied to control the Lorenz model. In [24], the tracking reference values were supposed to obey certain conditions, and this limited its ability to be applied in several real-world cases. In [25], the author suggested a new track control technique to avert the restrictions suggested in [24]. Using fewer sensors in a control strategy is preferred due to lower cost and simple realization.

In [25-27], an easy controller with output/state feedback was applied to control a general chaotic model. The studies in $[25,27,28]$ motivated us to propose the single-state feedback track synchronization control technique that is proved in our study here. Furthermore, lack of certainty cannot be avoided in real applications. Consequently, robust control has become a significant aspect of controller design $[25,28,29]$.

Although there is chaos in chaotic systems, they also have symmetrical features. In this research, we show that there is symmetry in the solutions of the chaotic system under study. In addition, synchronization between two chaotic systems means making them symmetrical to each other and this is the main aim of our proposed control strategy. Complete synchronization between two chaotic systems means complete symmetry between them, but phase synchronization means complete symmetry with a phase shift. In this study, based on a genetic algorithm (GA), a novel robust single-state feedback tracking synchronization control of two identical chaotic systems will be proposed. For an alternative approach, the gradient-based (or mathematical programming) method for solving differential-algebraic equation systems, interested readers can refer to [30] and the references cited there.

The main aim of this work is to design a new robust scheme by which two important types of chaotic synchronization (i.e., chaotic complete synchronization and chaotic phase synchronization) can be achieved based on a single-state feedback track synchronization control algorithm using the same controller. In addition, the robustness of the proposed controller is proved using a genetic algorithm. 
A new secure communication plan is also developed relying on the proposed robust single-state feedback track synchronization method as an application. The track control method is applied only to control chaotic systems [25]. To the best of our knowledge, this is the first time it has been applied in chaos synchronization and it is the first time two types of synchronization using the same controller have been achieved. In addition, phase synchronization is accomplished via a single-state feedback track controller that is less costly than feeding back all system states.

The paper is designed as follows. Following the current section, a novel single-state feedback track synchronization control algorithm is suggested in Section 2. The proposed algorithm is applied to the synchronization of two identical Lorenz chaotic systems in Section 3. A robust single-state feedback track synchronization optimal control design is produced in Section 4. As an application, the proposed robust track synchronization controller is applied to the development of a secure communication scheme in Section 5. Intensive simulation experiments are also presented in Sections 3-5. Our conclusions are presented in Section 6.

\section{Track Synchronization Algorithm}

In this section, we propose an algorithm by which the complete and phase synchronization can be done in the same structure (see Figure 1). The main idea of it is to give reference values for the synchronization errors. If the reference values of the synchronization errors are all zero then a complete synchronization is achieved, but if their values are not equal to zero a phase synchronization is achieved. All previous studies on the phase synchronization were done depending on the stability theory only, which needs to feedback all system states, but here we achieve the phase synchronization based on the proposed single-state feedback track synchronization control algorithm.

Let the states of the $n$-dimensional master and slave systems be denoted by $x_{i}$ and $y_{i}+u_{i}$, respectively, where $u_{i}$ 's are the control signals. The synchronization errors are denoted by $w_{i}=y_{i}+u_{i}-x_{i}$. This means that we have three systems: master, slave and synchronization errors systems. Our task is to control the synchronization errors system by proposing a controller such that the synchronization errors track predetermined reference values $w_{i r}$.

- $\quad$ Construct the synchronization errors system $w_{i}=y_{i}-x_{i}$.

- Construct the track synchronization control signals $u_{i}$.

- Construct the track synchronization control errors system $e_{i}=w_{i}-w_{i r}$.

- Apply stability criteria to calculate the range of the controller such that the track errors system is asymptotically stable.

- If the reference values $w_{i r}=0$ then a complete synchronization will be reached while a phase synchronization is accomplished if $w_{i r}=y_{i}(0)-x_{i}(0)$.

Let the master system be denoted by $d x_{i} / d t=f_{i}\left(x_{i}\right)$ and the slave synchronized system be denoted by $d y_{i} / d t=f_{i}\left(y_{i}\right)+u_{i}$. Then the synchronization errors system can be constructed as

$$
d w_{i} / d t=f_{i}\left(y_{i}\right)+u_{i}-f_{i}\left(x_{i}\right)=g_{i}\left(w_{i}\right)+u_{i}
$$

where $w_{i}=y_{i}-x_{i}$.

The track controller is proposed in such a way that feeding back only one state variable reduces the number of sensors. Assuming that the only measurable state is $w_{h}$, then the proposed controller takes the form

$$
u_{i}= \begin{cases}k\left(w_{h}-w_{h r}\right)-f_{h}\left(w_{h r}\right) & \text { if } i=h, \\ -f_{i}\left(w_{i r}\right) & \text { otherwise, }\end{cases}
$$

where $k$ is the gain of the closed loop track synchronization controller.

By putting $w_{i}=w_{i r}+e_{i}$ in the (1), the track error system can be constructed as

$$
d e_{i} / d t=H\left(x, y, k, w_{r}\right)
$$


The main task after that is to calculate the value of the controller gain $k$ that ensures that the track synchronization control errors system (3) is asymptotically stable.

In the next section, we apply this proposed method to the synchronization of two identical Lorenz systems.

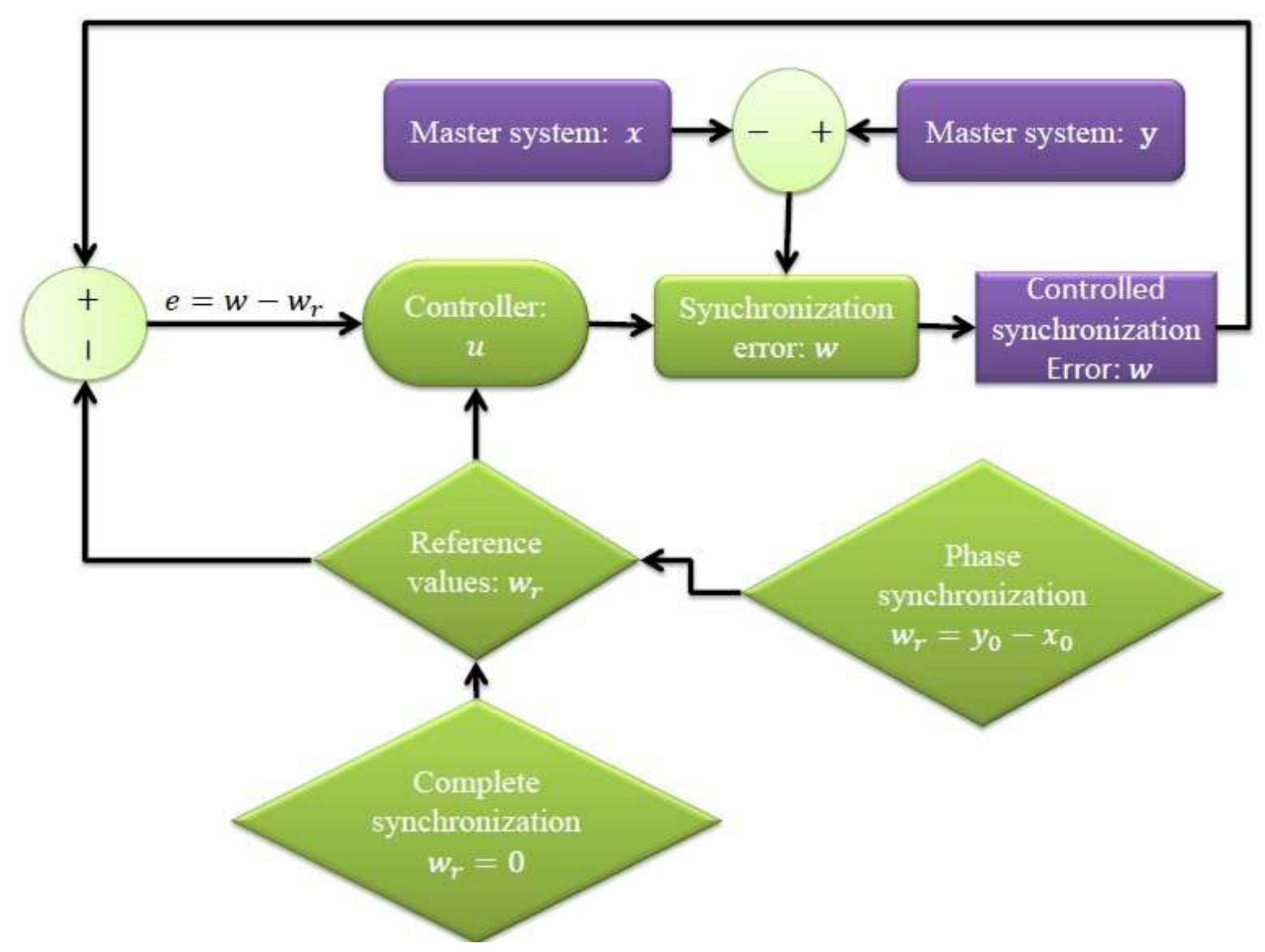

Figure 1. Block-diagram of tracking synchronization of two identical chaotic systems.

\section{Implementation of the Novel Track Synchronization Controller}

In this section, we apply the proposed algorithm to synchronize two identical Lorenz chaotic systems via two types of synchronizations (i.e., complete and phase synchronization) by the same single-state feedback controller which has not been applied before in the literature.

The master and slave systems are selected to be two identical Lorenz systems in the following forms:

$$
\begin{aligned}
& \frac{d x_{1}}{d t}=-\sigma x_{1}+\sigma x_{2}, \\
& \frac{d x_{2}}{d t}=r x_{1}-x_{2}-x_{1} x_{3} \\
& \frac{d x_{3}}{d t}=x_{1} x_{2}-b x_{3} .
\end{aligned}
$$


And

$$
\begin{aligned}
& \frac{d y_{1}}{d t}=-\sigma y_{1}+\sigma y_{2}+u_{1}, \\
& \frac{d y_{2}}{d t}=r y_{1}-y_{2}-y_{1} y_{3}+u_{2} \\
& \frac{d y_{3}}{d t}=y_{1} y_{2}-b y_{3}+u_{3} .
\end{aligned}
$$

where the parameters are selected here to be $(\sigma, b, r)=(10,8 / 3,28)$.

Figure 2 shows the time history of the master and the slave Lorenz chaotic models, where the initial conditions are $x_{0}=(10,5,-15)$ and $y_{0}=(1,1,-1)$. The difference between the two systems is clear due to the difference in their initial values.

Let the synchronization errors system be $w_{1}=y_{1}-x_{1}, w_{2}=y_{2}-x_{2}$ and $w_{3}=y_{3}-x_{3}$. Then the synchronization errors system can be represented as follows:

$$
\begin{aligned}
\frac{d w_{1}}{d t} & =-\sigma w_{1}+\sigma w_{2}+u_{1}, \\
\frac{d w_{2}}{d t} & =r w_{1}-w_{2}-x_{3} w_{1}-y_{1} w_{3}+u_{2} \\
\frac{d w_{3}}{d t} & =x_{1} w_{2}+y_{2} w_{1}-b w_{3}+u_{3} .
\end{aligned}
$$

Our task here is to control the synchronization errors system using the single-state feedback track control strategy that has not been used before for this purpose. This method enables us to control the synchronization errors to track any predetermined values $w_{i r}$ (reference values) for all $i \in\{1,2,3\}$.

Assume that the only measurable state in the system (6) is $w_{2}$, then it is the only one that will be used to construct the closed-loop single-state feedback track control as follows.

The single-state feedback track controller is proposed to be

$$
\begin{aligned}
& u_{1}=\sigma w_{1 r}-\sigma w_{2 r}, \\
& u_{2}=k\left(w_{2}-w_{2 r}\right)-r w_{1 r}+w_{2 r}+x_{3} w_{1 r}+y_{1} w_{3 r}, \\
& u_{3}=-x_{1} w_{2 r}-y_{2} w_{1 r}+b w_{3 r} .
\end{aligned}
$$

Figure 3 shows the control signals used to derive the synchronization error to follow its selected reference values.

Note that $w_{2}$ is the only state variable in the synchronization error system which is utilized to formulate the proposed closed-loop feedback and $k$ is the gain of the proposed track controller with single-state feedback.

The single-state feedback closed-loop controlled synchronization error system becomes

$$
\begin{aligned}
\frac{d w_{1}}{d t} & =-\sigma w_{1}+\sigma w_{2}+u_{1} \\
\frac{d w_{2}}{d t} & =r w_{1}-w_{2}-x_{3} w_{1}-y_{1} w_{3}+u_{2} \\
\frac{d w_{3}}{d t} & =x_{1} w_{2}+y_{2} w_{1}-b w_{3}+u_{3} .
\end{aligned}
$$


Let $e_{1}=w_{1}-w_{1 r}, e_{2}=w_{2}-w_{2 r}, e_{3}=w_{3}-w_{3 r}$. Substituting $w_{1}=w_{1 r}+e_{1}, w_{2}=w_{2 r}+e_{2}$, $w_{3}=w_{3 r}+e_{3}$ and (7) in (8), the tracking error dynamics of the synchronization error system (6) can be represented as follows:

$$
\begin{aligned}
& \frac{d e_{1}}{d t}=-\sigma e_{1}+\sigma e_{2}, \\
& \frac{d e_{2}}{d t}=k e_{2}+r e_{1}-e_{2}-x_{3} e_{1}-y_{1} e_{3}, \\
& \frac{d e_{3}}{d t}=x_{1} e_{2}+y_{2} e_{1}-b e_{3} .
\end{aligned}
$$

It is obvious that the point $E_{0}=(0,0,0)$ is an equilibrium point of the tracking error system (9) whose Jacobian matrix is calculated and can be written as follows:

$$
\left[\begin{array}{ccc}
-\sigma & \sigma & 0 \\
r-x_{3} & k-1 & -y_{1} \\
y_{2} & x_{1} & -b
\end{array}\right]
$$

Our task here is to find the range of synchronization controller gain $k$ that guarantees the stability of all Jacobian matrix (10) eigenvalues, which implies that the tracking synchronization control error dynamics $e_{1}, e_{2}$ and $e_{3}$ will be stable at the equilibrium point $E_{0}=(0,0,0)$ asymptotically. As a result the synchronization errors $w_{1}, w_{2}$ and $w_{3}$ will track their references $w_{1 r}, w_{2 r}$ and $w_{3 r}$ respectively. The main task is to determine an appropriate control gain $k$. Our result is recorded in Theorem 1 as follows.
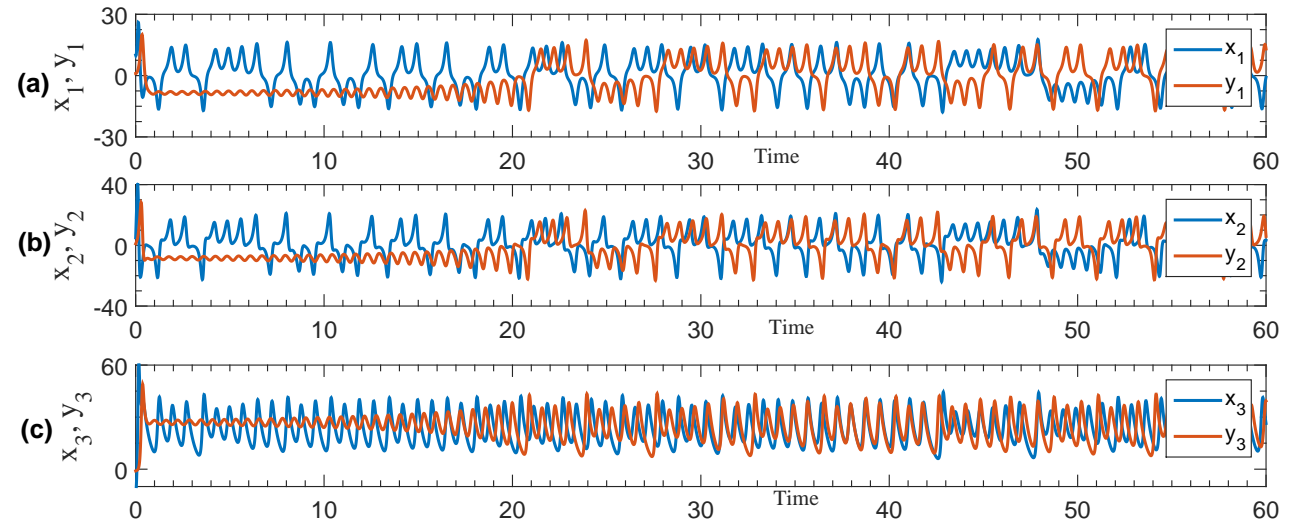

Figure 2. Dynamics of master $(x)$ and slave $(y)$ Lorenz chaotic systems where $x_{0}=(10,5,-15)$, $y_{0}=(1,1,-1)$ and $(\sigma, r, b)=(10,28,8 / 3)$.
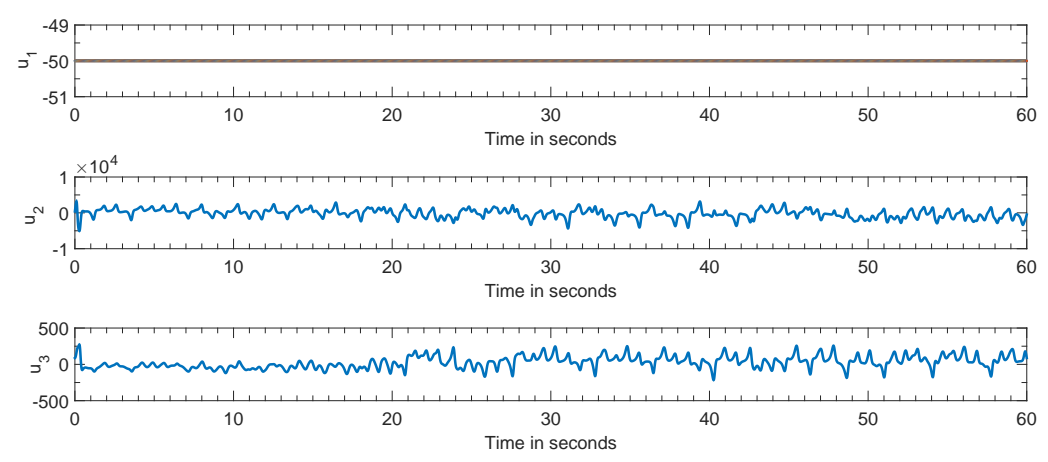

Figure 3. Proposed tracking synchronization control signals: $u_{1}, u_{2}, u_{3}$. 
Theorem 1. The synchronization error system (6) of the Lorenz synchronized systems (5) with the selected set of parameters via the tracking controller (7) can track any predetermined values $w_{1 r}, w_{2 r}$ and $w_{3 r}$ if the controller gain satisfies

$$
\begin{aligned}
k< & \min \left\{b+\sigma+1,\left(b \sigma+b+\sigma-\sigma\left(r-x_{3}\right)-x_{1} y_{1}\right) /(b+\sigma)\right. \\
& \left.-\left(b\left(\sigma\left(r-x_{3}\right)-\sigma\right)+\sigma\left(x_{1} y_{1}+y_{1} y_{2}\right)\right) / b \sigma, \frac{-B-\sqrt[2]{B^{2}-4 A C}}{2 A}\right\}
\end{aligned}
$$

where $A=(b+\sigma), B=\left(-2 \sigma-b^{2}-\sigma^{2}-2 b-2 b \sigma+r \sigma-\sigma x_{3}+x_{1} y_{1}\right)$ and $C=(b+\sigma+2 b \sigma-r \sigma+$ $\left.\sigma^{2}+\sigma x_{3}-x_{1} y_{1}+b \sigma^{2}+b^{2} \sigma-r \sigma^{2}+\sigma^{2} x_{3}+b^{2}-b x_{1} y_{1}+\sigma y_{1} y_{2}\right)$.

Proof. The Jacobian matrix characteristic Equation (10) is written as follows:

$$
a_{0} \lambda^{3}+a_{1} \lambda^{2}+a_{2} \lambda+a_{3}=0
$$

where

$$
\begin{gathered}
a_{0}=1, a_{1}=(b-k+\sigma+1), \\
a_{2}=\left(b(\sigma-k+1)-\sigma\left(r-x_{3}\right)-x_{1} y_{1}-\sigma(k-1)\right)
\end{gathered}
$$

and

$$
a_{3}=-\left(b\left(\sigma\left(r-x_{3}\right)+\sigma(k-1)\right)+x_{1} y_{1}(k-1)+x_{1} y_{1}(\sigma-k+1)+\sigma y_{1} y_{2}\right) .
$$

By applying the Routh-Hurwitz stability test [31], the eigenvalues of (11) have negative real parts if and only if $a_{0}=1>0 ; a_{1}=(b-k+\sigma+1)>0$, then

$$
k<b+\sigma+1
$$

$a_{2}=\left(b(\sigma-k+1)-\sigma\left(r-x_{3}\right)-x_{1} y_{1}-\sigma(k-1)\right)>0$, then

$$
k<\left(b \sigma+b+\sigma-\sigma\left(r-x_{3}\right)-x_{1} y_{1}\right) /(b+\sigma) ;
$$

$a_{3}=-\left(b\left(\sigma\left(r-x_{3}\right)+\sigma(k-1)\right)+x_{1} y_{1}(k-1)+x_{1} y_{1}(\sigma-k+1)+\sigma y_{1} y_{2}\right)>0$, then

$$
k<-\left(b\left(\sigma\left(r-x_{3}\right)-\sigma\right)+\sigma\left(x_{1} y_{1}+y_{1} y_{2}\right)\right) / b \sigma \Longrightarrow k \text { is negative; }
$$

$a_{1} a_{2}-a_{0} a_{3}>0$, then $k^{2}(b+\sigma)+k\left(-2 \sigma-b^{2}-\sigma^{2}-2 b-2 b \sigma+r \sigma-\sigma x_{3}+x_{1} y_{1}\right)+(b+\sigma+2 b \sigma-$ $\left.r \sigma+\sigma^{2}+\sigma x_{3}-x_{1} y_{1}+b \sigma^{2}+b^{2} \sigma-r \sigma^{2}+\sigma^{2} x_{3}+b^{2}-b x_{1} y_{1}+\sigma y_{1} y_{2}\right)>0$, then

$$
k<\frac{-B-\sqrt[2]{B^{2}-4 A C}}{2 A},
$$

where $A=(b+\sigma), B=\left(-2 \sigma-b^{2}-\sigma^{2}-2 b-2 b \sigma+r \sigma-\sigma x_{3}+x_{1} y_{1}\right)$ and $C=(b+\sigma+2 b \sigma-r \sigma+$ $\left.\sigma^{2}+\sigma x_{3}-x_{1} y_{1}+b \sigma^{2}+b^{2} \sigma-r \sigma^{2}+\sigma^{2} x_{3}+b^{2}-b x_{1} y_{1}+\sigma y_{1} y_{2}\right)$.

From (12) to (15), it easy to conclude that $k<\min \left\{b+\sigma+1,\left(b \sigma+b+\sigma-\sigma\left(r-x_{3}\right)-x_{1} y_{1}\right) /(b+\right.$ $\left.\sigma),-\left(b\left(\sigma\left(r-x_{3}\right)-\sigma\right)+\sigma\left(x_{1} y_{1}+y_{1} y_{2}\right)\right) / b \sigma, \frac{-B-\sqrt[2]{B^{2}-4 A C}}{2 A}\right\}$, which completes the proof.

Numerical Simulation of Tracking Synchronization of Two Identical Lorenz Systems

In this section, numerical simulation of the proposed tracking synchronization of two identical Lorenz systems is performed in order to demonstrate the above algorithm. Furthermore, we numerically solve systems (4) and (5) with parameters $(\sigma, b, r)=(10,8 / 3,28)$ and the initial conditions for the master and slave systems as $x_{0}=(10,5,-15)$ and $y_{0}=(1,1,-1)$, respectively. 
The numerical simulation of the proposed algorithm is executed for two cases of synchronization, namely chaotic complete synchronization and chaotic phase synchronization.

\section{Case 1. Complete synchronization}

The reference values are adjusted in this case as $\left(w_{1 r}, w_{2 r}, w_{3 r}\right)=(0,0,0)$. Figure 4a shows the synchronization errors system. It is clear that the synchronization errors approach zero in a short time. Figure $4 \mathrm{~b}$ shows the track synchronization control errors, from which it is obvious that the synchronization control errors tend to zero in a short time as predicted from the analytical results. In conclusion, from Figure 4, the chaotic complete synchronization is achieved in a short time.

(a)

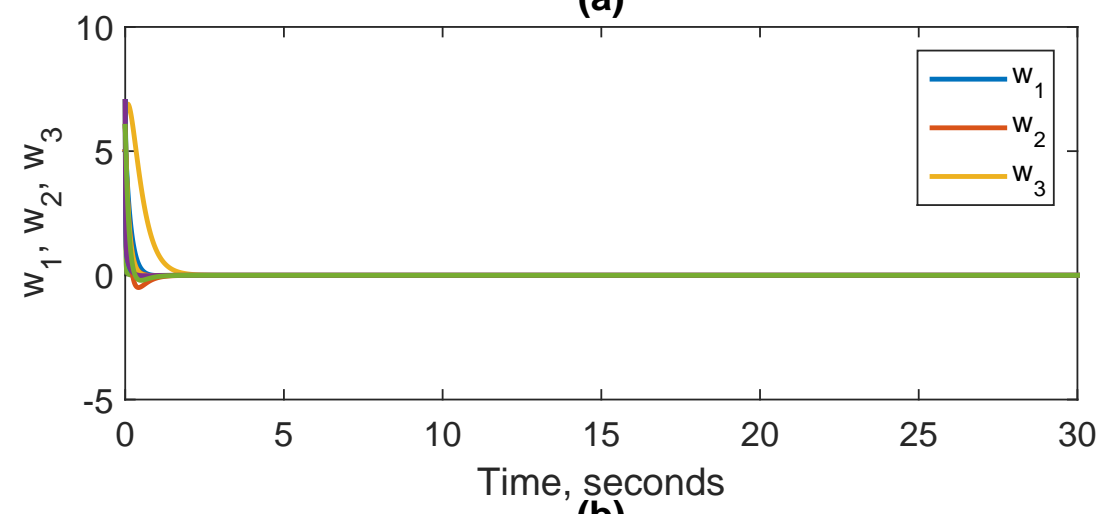

(b)

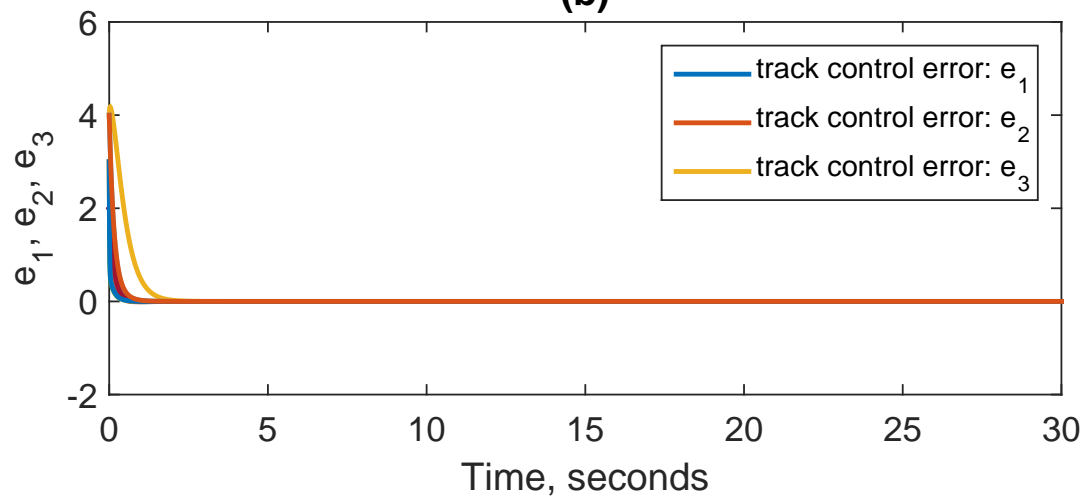

Figure 4. (a) Complete synchronization errors: $w_{i}=w_{i r}=0$, where $i=1,2$, 3. (b) Complete synchronization track control errors: $e_{1}, e_{2}, e_{3}$.

\section{Case 2. Phase synchronization}

In this case, the reference values are calculated as $\left(w_{1 r}, w_{2 r}, w_{3 r}\right)=\left(y_{10}-x_{10}, y_{20}-x_{20}, y_{30}-\right.$ $\left.x_{30}\right)=(-9,-4,14)$. This case is clearly different from the previous one. The reference values depend on the initial conditions of the two systems. It is clear from Figure 5a that the phase synchronization errors are tracking the predetermined reference values. The track synchronization control errors approach zero as shown in Figure $5 \mathrm{~b}$ as predicted from the analytical results. As a result, Figure 5 proves that the phase synchronization is accomplished quickly. 
(a)

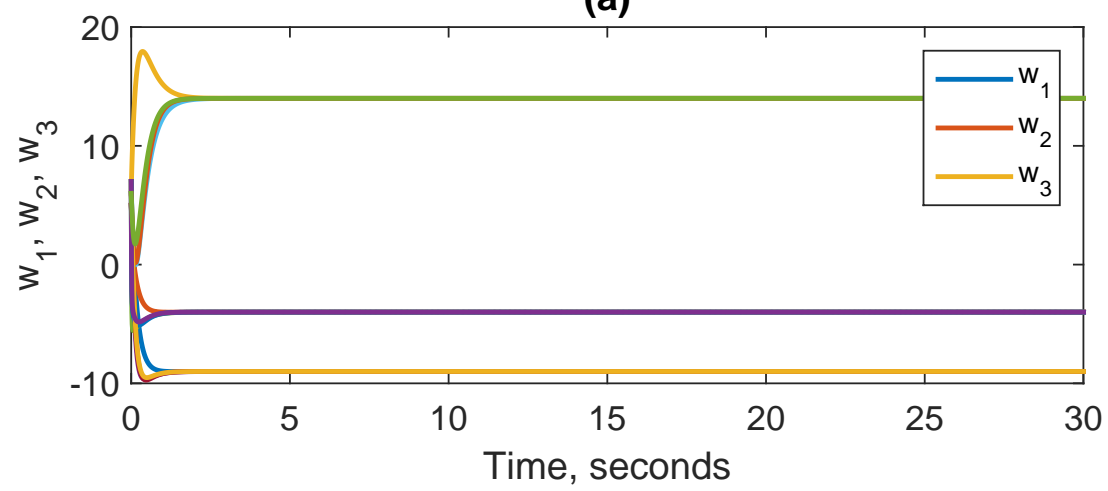

(b)

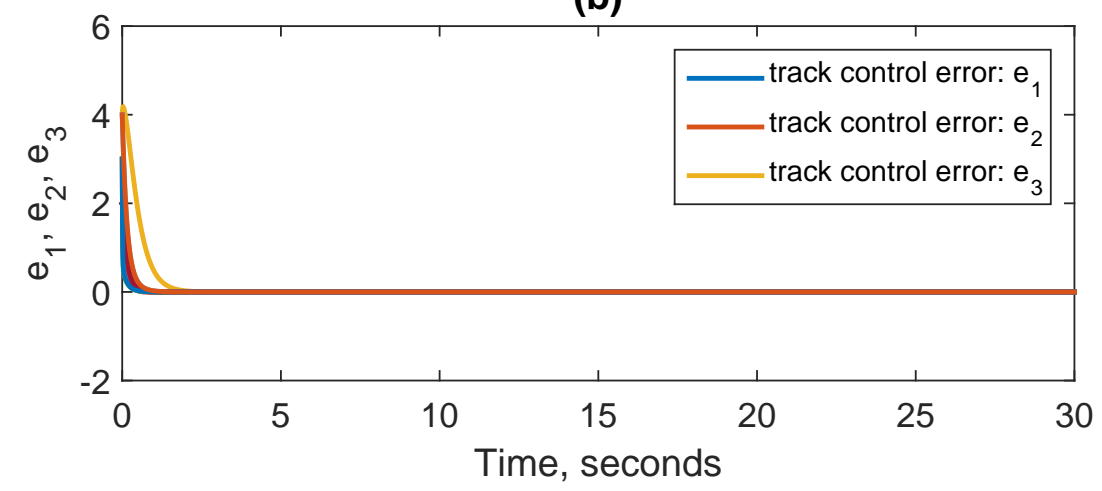

Figure 5. Phase synchronization errors and synchronization track control errors. (a) Phase synchronization errors: $w_{1}=w_{1 r}=y_{1}(0)-x_{1}(0)=-9, w_{2}=w_{2 r}=y_{2}(0)-x_{2}(0)=-4, w_{3}=$ $w_{3 r}=y_{3}(0)-x_{3}(0)=14$. (b) Synchronization track control errors: $e_{1}, e_{2}, e_{3}$.

In addition, in this case we will compute the phase difference and the amplitude of the master and slave systems numerically. The phase differences between the master and slave systems can be formed as $\phi_{m}^{x_{l}, x_{k}}-\phi_{s}^{y_{l}, y_{k}}, l, k=1,2,3$ and $l \neq k$, where $\phi_{m}^{x_{l}, x_{k}}=\tan ^{-1}\left(\frac{x_{k m}}{x_{l m}}\right)$ and $\phi_{s}^{y_{l}, y_{k}}=\tan ^{-1}\left(\frac{y_{k s}}{y_{l s}}\right)$ are phases of the master and slave systems, respectively. The phase differences $\phi_{m}^{x_{1}, x_{2}}-\phi_{s}^{y_{1}, y_{2}}$ and $\phi_{m}^{x_{1}, x_{3}}-\phi_{s}^{y_{1}, y_{3}}$ in $\left(x_{1}, x_{2}\right),\left(x_{1}, x_{3}\right)$ planes, respectively, are computed by and plotted in Figure $6 \mathrm{a}, \mathrm{b}$. It is clear the phase differences $\phi_{m}^{x_{1}, x_{2}}-\phi_{s}^{y_{1}, y_{2}}$ and $\phi_{m}^{x_{1}, x_{3}}-\phi_{s}^{y_{1}, y_{3}}$ are finite and fluctuate in a chaotic mode, which implies that CPS is accomplished. Figure 6c displays the relationship $\phi_{m}^{x_{1}, x_{2}}-\phi_{s}^{y_{1}, y_{2}}$ and $\phi_{m}^{x_{1}, x_{3}}-\phi_{s}^{y_{1}, y_{3}}$. As shown, this relationship is a chaotic attractor and this means that $\phi_{m}^{x_{1}, x_{2}}-\phi_{s}^{y_{1}, y_{2}}$ and $\phi_{m}^{x_{1}, x_{3}}-\phi_{s}^{y_{1}, y_{3}}$ change in chaotic style and CPS is active.

In addition, the master system amplitude in $\left(x_{1}, x_{2}\right)$ projection is formed as $A_{m}^{x_{1}, x_{2}}=$ $\sqrt{\left(x_{1}\right)^{2}+\left(x_{2}\right)^{2}}$, and the same is formed for the slave as $A_{s}^{y_{1}, y_{2}}=\sqrt{\left(y_{1}\right)^{2}+\left(y_{2}\right)^{2}}$. In Figure 7a,b, these amplitudes are graphed with respect to $t$, whilst Figure $2 \mathrm{c}$ displays $A_{s}^{y_{1}, y_{2}}$ versus $A_{m}^{x_{1}, x_{2}}$. Figure $7 \mathrm{c}$ shows that those amplitudes have a chaotic style and they are linearly independent (uncorrelated). Similar results can be found for the other phase variations and amplitude relationships for the master and slave models on different projection planes. 

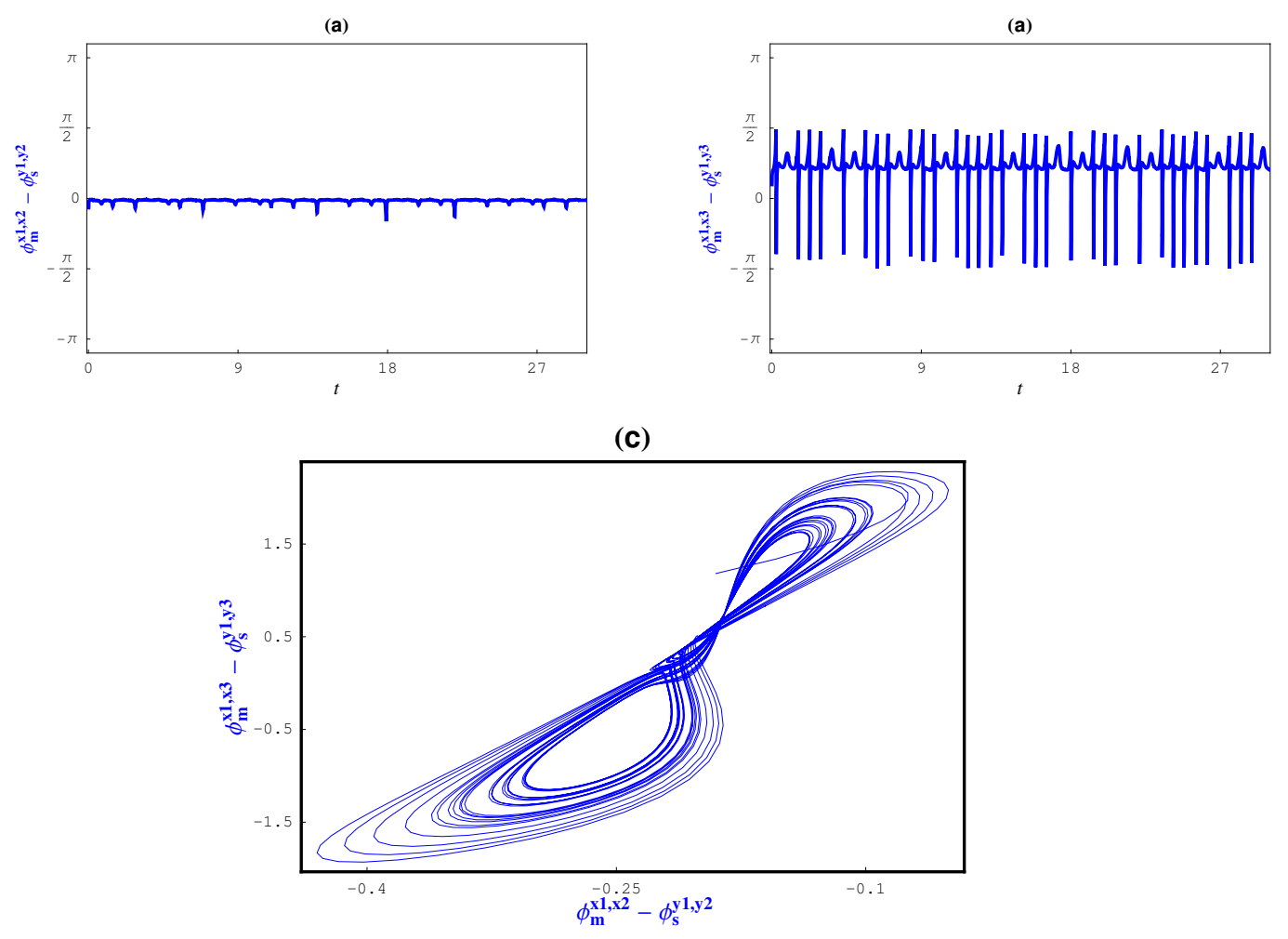

Figure 6. Phase differences of MPPS of models (4) and (5) are finite and can move in a chaotic style: (a) $\phi_{m}^{x_{1}, x_{2}}-\phi_{s}^{y_{1}, y_{2}}$ against $t$, (b) $\phi_{m}^{x_{1}, x_{3}}-\phi_{s}^{y_{1}, y_{3}}$ against $t$ and (c) the chaotic attractor of $\phi_{m}^{x_{1}, x_{2}}-\phi_{s}^{y_{1}, y_{2}}$ and $\phi_{m}^{x_{1}, x_{3}}-\phi_{s}^{y_{1}, y_{3}}$.
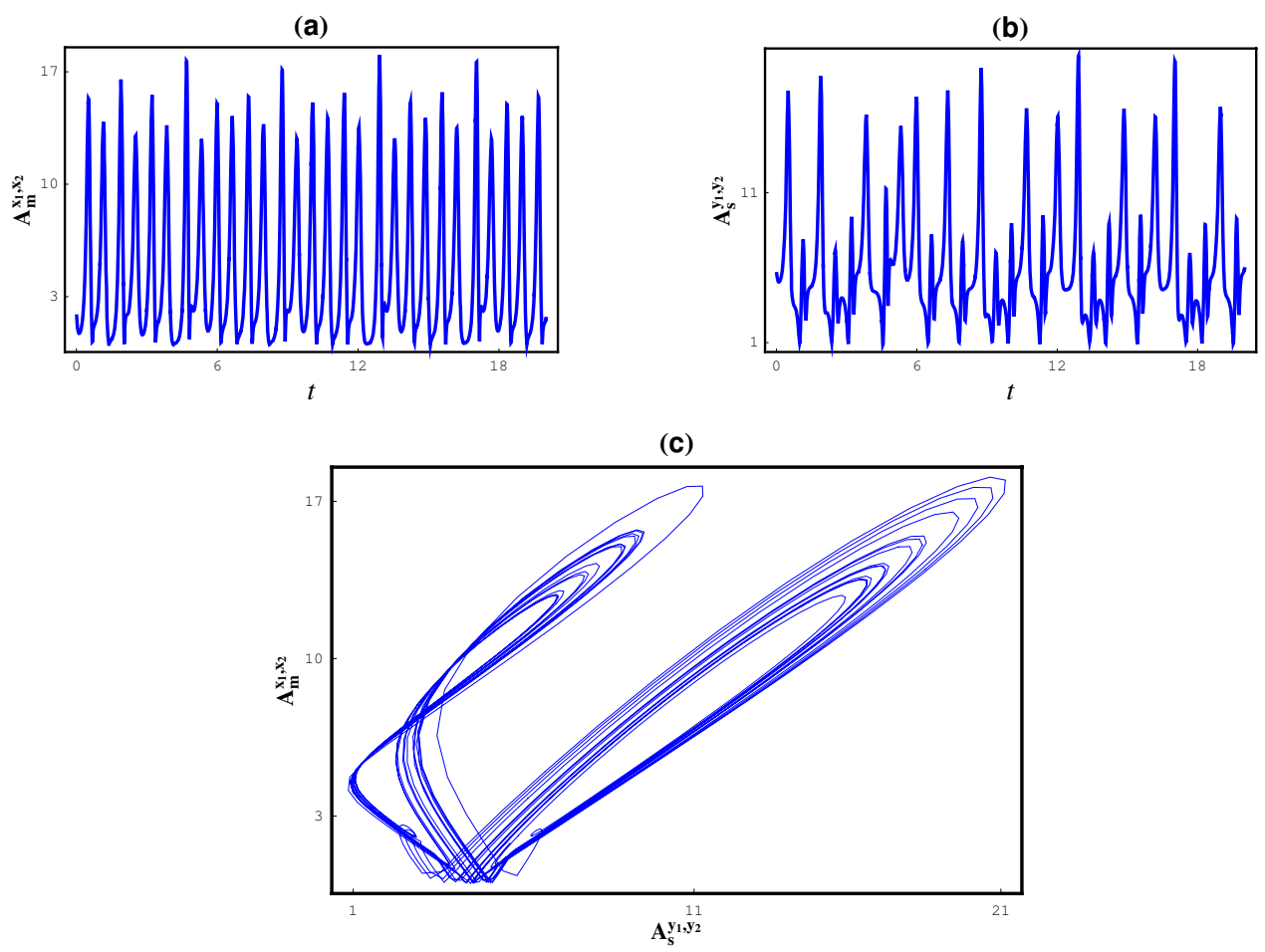

Figure 7. Amplitudes of models (4) and (5) in $\left(x_{1}, x_{2}\right)$ plane: (a) $A_{m}^{x_{1}, x_{2}}$ against $t$. (b) $A_{S}^{y_{1}, y_{2}}$ against $t$. (c) The relationship of $A_{m}^{x_{1}, x_{2}}$ and $A_{S}^{y_{1}, y_{2}}$. 


\section{Robust Track Synchronization for Two Identical Lorenz Chaotic Systems}

\subsection{Designing a Robust Tracking Synchronization Control Algorithm}

In a practical environment, there exist certain unavoidable uncertainties. Consequently, it is useful to consider robustness with respect to perturbations in track synchronization control if applied for two identical Lorenz systems. Suppose that the parameters of the model have some perturbations, the uncertain synchronization error system can be represented as

$$
\begin{aligned}
& \frac{d w_{1}}{d t}=-(\sigma+\Delta \sigma) w_{1}+(\sigma+\Delta \sigma) w_{2}+u_{1}, \\
& \frac{d w_{2}}{d t}=(r+\Delta r) w_{1}-w_{2}-x_{3} w_{1}-y_{1} w_{3}+u_{2}, \\
& \frac{d w_{3}}{d t}=x_{1} w_{2}+y_{2} w_{1}-(b+\Delta b) w_{3}+u_{3} .
\end{aligned}
$$

Let $d_{1}=\Delta \sigma\left(w_{2}-w_{1}\right), d_{2}=\Delta r w_{1}$ and $d_{3}=-\Delta b w_{3}$. Applying the designed controller whose form is represented in (7) and the model (16), the single-state feedback tracking synchronization control error of the the single-state feedback tracking synchronization error system becomes

$$
\begin{aligned}
& \frac{d e_{1}}{d t}=-\sigma e_{1}+\sigma e_{2}+d_{1}, \\
& \frac{d e_{2}}{d t}=k e_{2}+r e_{1}-e_{2}-x_{3} e_{1}-y_{1} e_{3}+d_{2}, \\
& \frac{d e_{3}}{d t}=x_{1} e_{2}+y_{2} e_{1}-b e_{3}+d_{3} .
\end{aligned}
$$

It can be rewritten in matrix form as

$$
d e / d t=A e+d
$$

where

$$
\begin{aligned}
d e / d t=\left[\begin{array}{l}
\frac{d e_{1}}{d t} \\
\frac{d e_{2}}{d t} \\
\frac{d e_{3}}{d t}
\end{array}\right], d=\left[\begin{array}{l}
d_{1} \\
d_{2} \\
d_{3}
\end{array}\right] \text { and } & \\
A & =\left[\begin{array}{ccc}
-\sigma & \sigma & 0 \\
r-x_{3} & k-1 & -y_{1} \\
y_{2} & x_{1} & -b
\end{array}\right] .
\end{aligned}
$$

The track control error of the synchronization error system is presented in the frequency-domain as $e(s)=(s I-A)^{-1} d(s)$.

For the robust controller our goal is to choose an appropriate value for the gain $k$ in order to minimize the consequences of disturbance $d$. From (18) and (19), the effects from $d_{2}$ can be decreased via choosing an appropriate value for the gain $k$, whilst the selected value of the gain $k$ has no impact to minimize the consequences of $d_{1}$ and $d_{2}$. As a result, we can improve the track synchronization controller as follows:

$$
\begin{aligned}
& u_{1}=k_{1}\left(w_{2}-w_{2 r}\right)+\sigma w_{1 r}-\sigma w_{2 r} \\
& u_{2}=k_{2}\left(w_{2}-w_{2 r}\right)-r w_{1 r}+w_{2 r}+x_{3} w_{1 r}+y_{1} w_{3 r} \\
& u_{3}=k_{3}\left(w_{2}-w_{2 r}\right)-x_{1} w_{2 r}-y_{2} w_{1 r}+b w_{3 r}
\end{aligned}
$$

where $k_{1}, k_{2}$ and $k_{3}$ are gains of the single-state feedback track synchronization controller. 
By utilizing (16) and (20), the closed-loop track synchronization control error dynamics for the synchronization errors system can be represented as follows:

$$
\begin{aligned}
& \frac{d e_{1}}{d t}=-\sigma e_{1}+\left(k_{1}+\sigma\right) e_{2}+p_{1}, \\
& \frac{d e_{2}}{d t}=\left(r-x_{3}\right) e_{1}+\left(k_{2}-1\right) e_{2}-y_{1} e_{3}+p_{2}, \\
& \frac{d e_{3}}{d t}=y_{2} e_{1}+\left(k_{3}+x_{1}\right) e_{2}-b e_{3}+p_{3} .
\end{aligned}
$$

That can be represented in compact matrix form as

$$
d e / d t=B e+p,
$$

where $e=\left[\begin{array}{l}e_{1} \\ e_{2} \\ e_{3}\end{array}\right]$

$$
B=\left[\begin{array}{ccc}
-\sigma & k_{1}+\sigma & 0 \\
r-x_{3} & k_{2}-1 & -y_{1} \\
y_{2} & k_{3+} x_{1} & -b
\end{array}\right]
$$

and $p=\left[\begin{array}{l}p_{1} \\ p_{2} \\ p_{3}\end{array}\right]$ where $p_{1}=\Delta \sigma\left(w_{2}-w_{1}\right), p_{2}=\Delta r w_{1}$ and $p_{3}=-\Delta b w_{3}$.

The Laplace transform of (22) is written as

$$
e(s)=(s I-B)^{-1} p(s)
$$

As a consequence of this, it is possible to select appropriate values of the gain $k_{1}, k_{2}$ and $k_{3}$ that decrease all disturbance effects. To do this, the following proposed fitness function can be minimized:

$$
f=\sum_{i}\left\|\left((s I-B)^{-1}\right)\right\|_{s_{i}=j \omega_{p_{i}}}
$$

where $w_{p_{i}}(i=1,2, \ldots)$ are the dominant frequencies related to the disturbances. We use the fast Fourier transform (FFT) [32] to determine the dominant frequencies of the $w_{2}=y_{2}-x_{2}$ (that are used for feeding back the tracking controller). Figure 8 shows the FFT of $w_{2}=y_{2}-x_{2}$. We choose the genetic algorithm to minimize the fitness function (24) because of its power as an optimization technique [33].

The optimization process for selecting robust optimal gains $k_{1}, k_{2}$ and $k_{3}$ is recorded as follows.

GA Track synchronization control procedure. Select optimal gains $k_{1}, k_{2}$ and $k_{3}$ using GA algorithm.

(1) Determine the dominant frequencies via FFT of the suggested measurable signal $w_{2}=y_{2}-x_{2}$.

(2) Design the fitness function as in (24).

(3) The nonlinear constraints are set up as $\operatorname{Re}\left(\lambda_{i}\right) \leq-\theta$, where $\left\{\lambda_{i} \mid \operatorname{det}\left(\lambda_{i} I-B\right)=0\right\}, i=1,2,3$, and $\theta$ is a chosen positive real angle.

(4) Utilize Matlab's GA toolbox to search for the optimal gains $\left(k_{1}, k_{2}, k_{3}\right)$.

\subsection{Numerical Simulation for Robust Track Synchronization Control}

Consider the master system (4) and the slave system (5) with $(\sigma, r, b)=(10,28,8 / 3)$ where $x_{0}=(10,5,-15)$ and $y_{0}=(1,1,-1)$. The disturbances are $p_{1}=\sin (2 \pi t)\left(w_{2}-w_{1}\right)+$ noise signal, $p_{2}=\sin (4 \pi t) w_{1}+$ noise signal, and $p_{3}=\sin (6 \pi t) w_{3}$, where the noise signal is a band-limited white 
noise signal. The spectrum of $w_{2}$ can be obtained by FFT as shown in Figure 8 . The dominant frequencies are selected as $f r_{1}=0.01 ; f r_{2}=0.02 ; f r_{3}=0.08 ; f r_{4}=0.1 ; f r_{5}=0.16 ; f r_{6}=0.24$; $f r_{7}=0.26$. Consequently, $j \omega_{p_{i}}=2 \pi f_{i}$, where $i=1,2,3,4,5,6,7$ are utilized for computing the fitness function (24).

Remark 1. The white noise is computed using the standard deviation of the feedbacked state as ss $=\operatorname{std}\left(y_{2}-\right.$ $\left.x_{2}\right)$. Then compute $n$ such that the signal to noise ratio $s n r=20 * \log 10(s / n)$. Give a snr value and compute $n$, then compute the white noise as noise $=n *(\operatorname{randn}(:, 1))$.

Assume that $\theta=2$ and set the population size and the number of generation equal to 100 . The execution of GA optimization is shown in Figure 9. The GA produces the following optimal gains.

$$
\left(k_{1}, k_{2}, k_{3}\right)=(-0.05,-200,-1),
$$

and the best fitness value is 168.8316 .

Using the optimal gains (25), the tracking synchronization robust controller error dynamics are depicted in Figures 10 and 11. Other testing sets of gain values are used for more testing where $\left(k_{1}, k_{2}, k_{3}\right)=(-1,-100,-2)$.

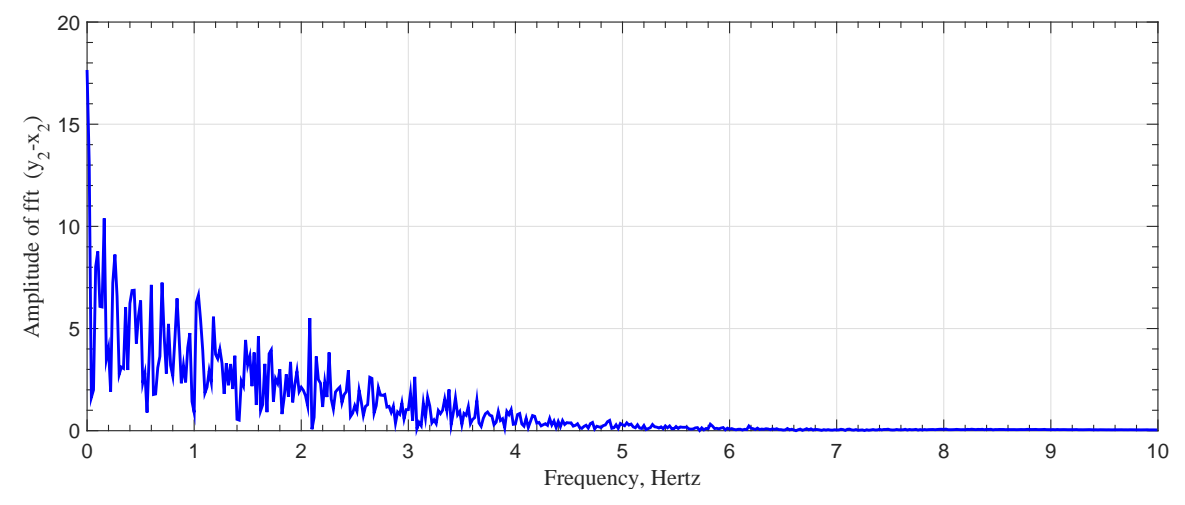

Figure 8. Fast Fourier transform of the closed loop feedbacked signal $w_{2}$.

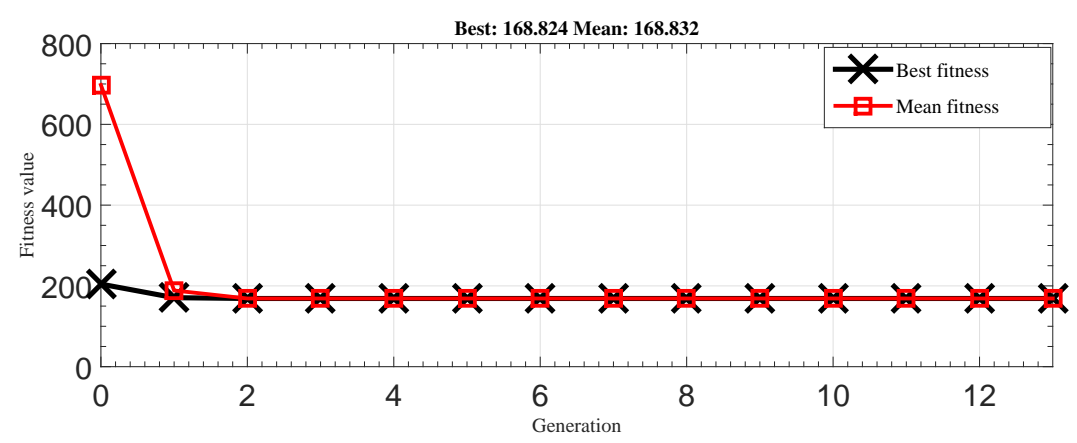

Figure 9. Genetic algorithm optimization. 


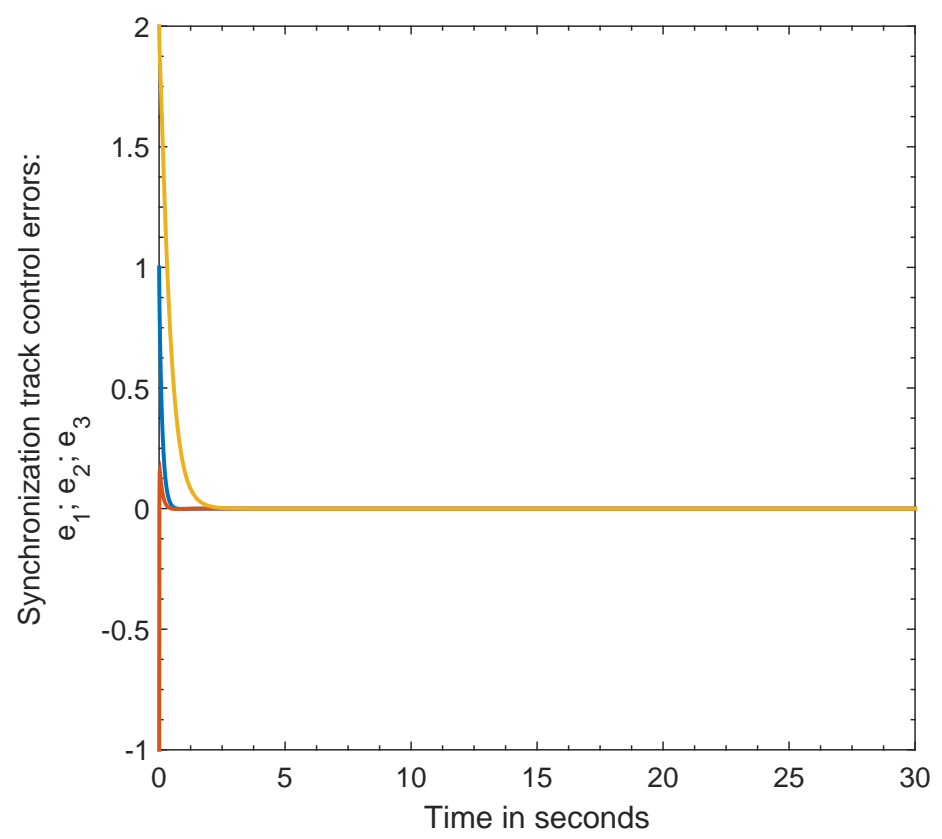

Figure 10. Synchronization robust track control errors where synchronization track controller gains are $k_{1}=-0.05 ; k_{2}=-200 ; k_{3}=-1$.

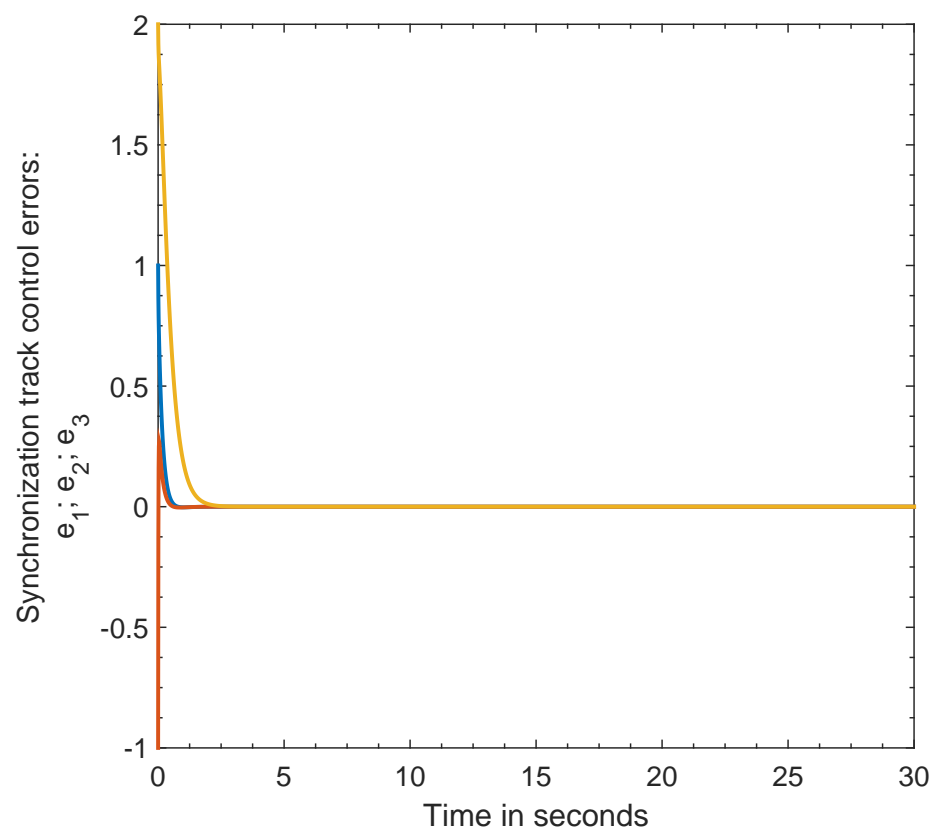

Figure 11. Synchronization robust track control errors where synchronization track controller gains are $k_{1}=-1 ; k_{2}=-100 ; k_{3}=-2$.

\section{Secure Communication Application via Robust Track Synchronization Control Results}

Secure communication is an important application of the topic and these types of synchronization have been studied in the literature [34-36]. In this section we will take advantage of the results of the robust synchronization that we obtained in order to provide a secure communication application. In Figure 12, we propose a scheme for complete robust synchronization secure communications. As is clear from this scheme, we consider the master and slave systems as transmitter and receiver 
systems, respectively. The most important thing that distinguishes this scheme from previous plans is the presence of noise when the message is sent from the sending equipment to the receiving equipment, and despite this, the message extraction process occurs successfully. Let the signal of the transmitted data message be denoted by $r(t)$ and let $\Theta=\Phi\left(r, x_{1}, x_{2}, x_{3}\right)$ be a complex invertible mapping that is used to encrypt the signals of the transmitter system in a chaotic manner. Then, combine the encrypted signal $\Theta$ with one (or more) of the three state variables, with any pair of their products or with their product. For illustration purposes, after adding this to the state variable $x_{3}$ the encrypted message becomes $\Gamma(t)=\Theta+x_{3}$. After that, the chaotic carrying signal of the sender device is produced and the encrypted message is transferred to the recipient end. When the message is sent there is strong noise that will intercept the message as well as the signals of the transmitter system. Therefore, if the synchronization used in making this application is not robust, this could affect message extraction. At the receiver side, we construct the controller as proposed in Equation (20). As a result, the synchronization between the drive (master) (4) and the identical controlled (5) frameworks will occur after a certain time $t_{s}$ and the difference between the master system $x$ and the slave system states $y$ will be zero in a specific period $t_{c}$ that is a time period longer than the time period $t_{s}\left(t_{s}\right.$ is the time period after which the synchronization process starts). For illustration purposes take $t_{c}=5 \mathrm{~s}$, recovering $\Gamma(t)$ via a straightforward mutation $\Theta=\Gamma(t)-y_{3}$ that starts at the receiver end. At the end, the sent data can be retrieved via an inverting operation $r^{*}(t)=\Phi^{-1}\left(y_{1}, y_{2}, y_{3}, \Theta\right)$ because the nonlinear function $\Phi$ is invertible.

The outputs of the numerical experiments are given in the following lines, where the values of the transmitter framework and receiver framework are adjusted to be as per those in Section 3 . For illustration purposes, the invertible function is selected as $\Theta=x_{1}+r(t)$ and $r(t)$ is taken for example as $r(t)=\sin (t) \cos (5 t)+1$. Suppose that the encrypted signal $\Theta$ is combined with the state variable $x_{3} \Longrightarrow \Gamma(t)=x_{1}+r(t)+x_{3}$. Figure 13 displays the results of secure communication via chaos synchronization. The sent message $r(t)$ and the transferred chaos signal $\Gamma(t)$ are, respectively, displayed in Figure 13a,b. Figure 13c displays the recovered message $r^{*}(t)=\Gamma(t)-y_{1}-y_{3}$. Figure 13d displays the error between the authentic sent message and the data signal of the retrieved message. It is clear from Figure 13d, that the sent message data signal $r(t)$ is retrieved exactly after a short transitory time.
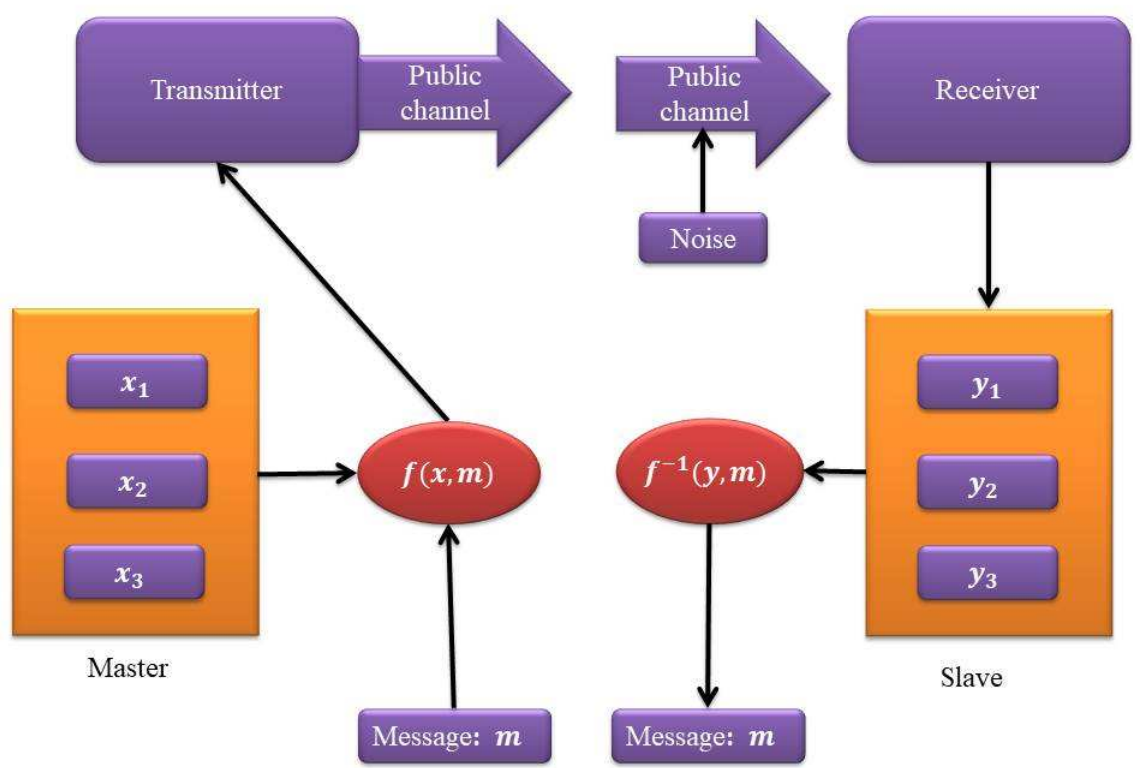

Figure 12. Proposed secure communication schematic diagram. 

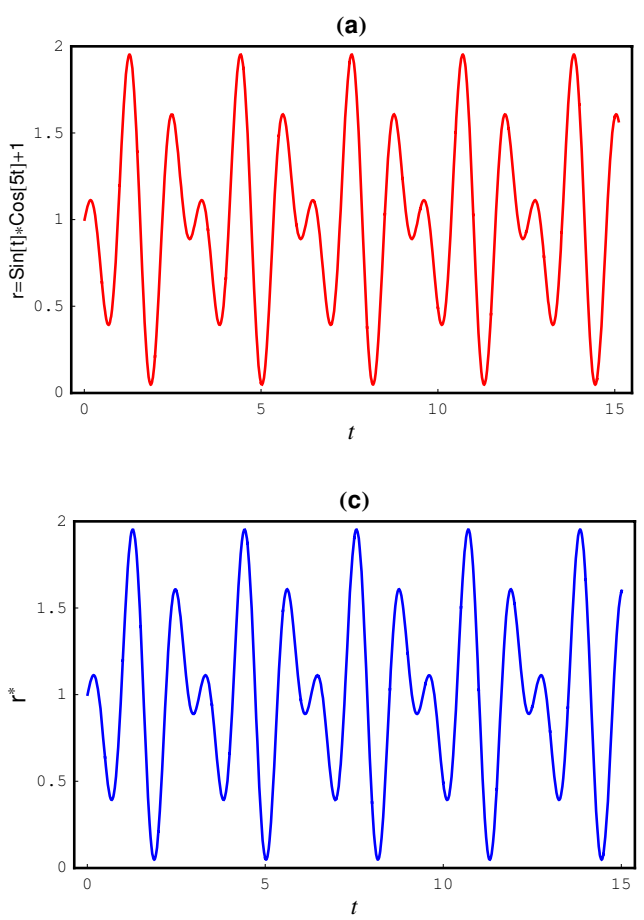

(b)

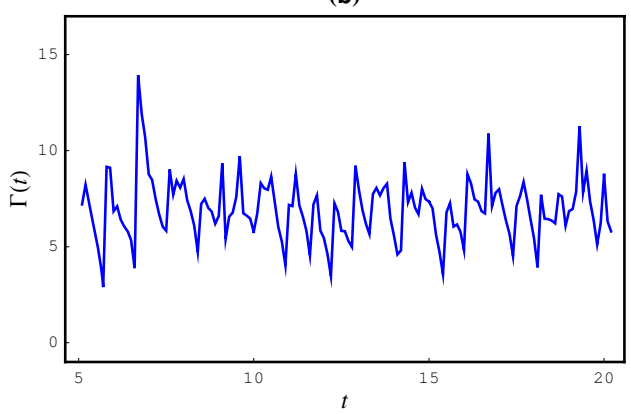

(d)

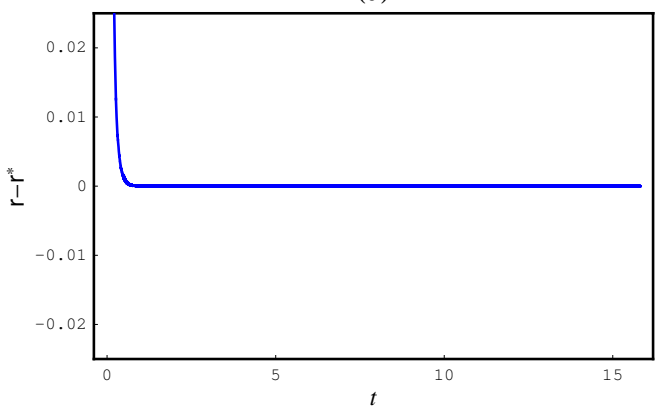

Figure 13. Secure communication simulation results. (a) $r(t)$ is the authentic message. (b) $\Gamma(t)$ is the transferred signal. (c) $r^{*}(t)$ is the retrieved message. (d) $r(t)-r^{*}(t)$ is the error signal.

\section{Conclusions}

This study has been suggested to accomplish two types of synchronization between the master and the controlled slave Lorenz chaotic systems via a single-state feedback track controller. In addition, the robustness of the proposed scheme has been proved and the gains of the proposed controller have been determined based on a genetic algorithm. Furthermore, the proposed scheme has been successfully applied to a secure communication system. The experimental results verify that the methods are correct and practical. Finally, we can confirm that this study has made the following contributions.

1. Design of a single-state feedback track synchronization controller to accomplish two types of synchronization between two identical Lorenz chaotic systems.

2. The complete and phase synchronizations of two identical Lorenz chaotic systems are achieved via the same controller, which has not been done before.

3. The single-state feedback strategy is less costly and its realization is simple since it uses fewer sensors.

4. The powerful GA algorithm has been combined with our proposed strategy to compute the suitable gains of the proposed synchronization controller.

5. More complex chaotic systems will likely require more complex treatment of parameters to fit between systems to achieve a decent measure of synchronization. This can be seen from the condition in Theorem 1 required to measure the degree of synchronization.

6. The proposed method has been successfully applied in a secure communication application.

Author Contributions: Conceptualization: E.E.M. and M.H. Data curation: E.E.M. and M.H. Formal analysis: E.E.M., M.H. and O.A.A. Funding acquisition: E.E.M. Investigation: E.E.M. and M.H. Methodology: E.E.M. and M.H. Project administration: E.E.M. Resources: E.E.M. and M.H. Software: E.E.M. and M.H. Supervision: E.E.M. Validation: E.E.M. and M.H. Visualization: E.E.M. and M.H. Writing-original draft: E.E.M. and M.H. Writing-review and editing: E.E.M., M.H. and O.A.A. All authors have read and agreed to the published version of the manuscript. 
Funding: This paper was funded by “Taif University Researchers Supporting Project number (TURSP-2020/20), Taif University, Taif, Saudi Arabia".

Acknowledgments: Taif University Researchers Supporting Project number (TURSP-2020/20), Taif University, Taif, Saudi Arabia.

Conflicts of Interest: The authors declare that they have no conflicts of interest.

\section{References}

1. Lv, X.; Cao, J.; Li, X.; Abdel-Aty, M.; Al-Juboori, U.A. Synchronization Analysis for Complex Dynamical Networks With Coupling Delay via Event-Triggered Delayed Impulsive Control. IEEE Trans. Cybern. 2020, 1-10. [CrossRef] [PubMed]

2. Shi, Y.; Cao, J. Finite-time synchronization of memristive Cohen-Grossberg neural networks with time delays. Neurocomputing 2020, 377, 159-167. [CrossRef]

3. Anischenko, V.; Vadivasova, T.; Postnov, D.; Safanova, M. Synchronization of chaos. Int. J. Bifur. Chaos 1992, 2, 633-644. [CrossRef]

4. Pikovsky, A.S.; Rosenblum, M.G.; Osipov, G.V.; Kurths, J. Phase synchronization of chaotic oscillators by external driving. Physica D 1997, 104, 219-238. [CrossRef]

5. Rosenblum, M.G.; Pikovsky, A.S.; Kurths, J. Phase synchronization of chaotic oscillators. Phys. Rev. Lett. 1996, 76, 1804-1807. [CrossRef] [PubMed]

6. Rosa, E.; Pardo, W.B.; Ticos, C.M.; Walkenstein, J.A.; Monti, M. Phase synchronization of chaos in a plasma discharge tube. Int. J. Bifur. Chaos 2000, 10, 2551-2563. [CrossRef]

7. Tallon-Baudry, C.; Bertrand, O.; Delpuech, C.; Pernier, J. Stimulus specificity of phase-locked and non-phase-locked $40 \mathrm{~Hz}$ visual responses in human. J. Neurosci. 1996, 16, 4240-4249. [CrossRef]

8. Wang, W.; Kiss, I.Z.; Hudson, J.L. Experiments on arrays of globally coupled chaotic electrochemical oscillators: Synchronization and clustering. Chaos 2000, 10, 248-256. [CrossRef]

9. Dabrowski, A.; Galias, Z.; Ogorzalek, M. Observations of phase synchronization phenomena in one-dimensional arrays of coupled chaotic electronic circuits. Int. J. Bifurc. Chaos 2000, 10, 2391-2398. [CrossRef]

10. Mahmouda, E.E.; Eshmawi, A.A. Secure communication and synchronizations in light of the stability theory of the hyperchaotic complex nonlinear systems. J. Intell. Fuzzy Syst. 2020, 38, 2569-2583. [CrossRef]

11. Pikovsky, A.S.; Rosenblum, M.G.; Kurths, J. Synchronization: A Universal Concept in Nonlinear Sciences; Cambridge University Press: Cambridge, UK, 2003.

12. Mahmoud, G.M.; Mahmoud, E.E. Phase and antiphase synchronization of two identical hyperchaotic complex nonlinear systems. Nonlinear Dyn. 2010, 61, 141-152. [CrossRef]

13. Mahmoud, E.E. Dynamics and synchronization of new hyperchaotic complex Lorenz system. Math. Comput. Model. 2012, 55, 1951-1962. [CrossRef]

14. Mahmoud, E.E.; Abo-Dahab, S.M. Dynamical properties and complex anti synchronization with applications to secure communications for a novel chaotic complex nonlinear model. Chaos Solitons Fractals 2018, 106, 273-284. [CrossRef]

15. Mahmoud, E.E.; Higazy, M.; Al-Harthi, T.M. A new nine-dimensional chaotic Lorenz system with quaternion Variables: Complicated dynamics, electronic circuit design, anti-anticipating synchronization, and chaotic masking communication application. Mathematics 2019, 7, 877. [CrossRef]

16. Milnor, J. On the concept of attractor. Commun. Math. Phys. 1985, 99, 177-195. [CrossRef]

17. Li, Y.; Li, H.; Ding, X.; Zhao, G. Leader-follower consensus of multiagent systems with time delays over finite fields. IEEE Trans. Cybern. 2019, 49, 3203-3208. [CrossRef]

18. Ott, E.; Sommerer, J.C. Blowout bifurcations-the occurrence of riddled basins and on-off intermittency. Phys. Lett. A 1994, 188, 39-47. [CrossRef]

19. Ashwin, P.; Buescu, J.; Stewart, I. Bubbling attractors and synchronization of chaotic oscillators Phys. Lett. A 1994, 193, 126-139. [CrossRef]

20. Grebogi, C.; Ott, E.; Yorke, J.A. Basin boundary metamorphoses: Changes in accessible boundary orbits. Physica D 1987, 24, 243-262. [CrossRef]

21. Li, H.L.; Jiang, H.; Cao, J. Global synchronization of fractional-order quaternion-valued neural networks with leakage and discrete delays. Neurocomputing 2019, 30, 211-219. [CrossRef] 
22. Cuomo, K.; Oppenheim, A.; Strogatz, S. Synchronization of Lorenz-based chaotic circuits with applications to communications. IEEE Trans. Circuits Syst. II 1993, 40, 626-633. [CrossRef]

23. Chen, H.; Liau, B.; Hou, Y. Hardware implementation of Lorenz circuit systems for secure chaotic communication applications. Sensors 2013, 13, 2494-2505. [CrossRef]

24. Yang, S.; Chen, C.; Yau, H. Control of chaos in Lorenz system. Chaos Solitons Fractals 2002, 13, 767-780. [CrossRef]

25. Gao, R. A novel track control for Lorenz system with single state feedback. Chaos Solitons Fractals 2019, 122, 236-244. [CrossRef]

26. Cheng, D.; Qi, H.; Li, Z. Analysis and Control of Boolean Networks: A Semi-Tensor Product Approach; Springer: London, UK, 2011.

27. Shen, Z.; Li, J. Chaos control for unified chaotic systems using output feedback controllers. Math. Comput. Simul. 2017, 132, 208-219. [CrossRef]

28. Pratap, A.; Raja, R.; Agarwal, R.P.; Cao, J. Stability analysis and robust synchronization of fractional-order competitive neural networks with different time scales and impulsive perturbations. Int. J. Adapt. Signal Process. 2019, 33, 1635-1660. [CrossRef]

29. Zuñiga-Aguilar, C.; Gómez-Aguilar, J.; Escobar-Jiménez, R.; Romero-Ugalde, H. Robust control for fractional variable-order chaotic systems with non-singular kernel. Eur. Phys. J. Plus 2018, 133, 1-13. [CrossRef]

30. Cuthrell, J.E.; Biegler, L.T. On the optimization of differential-algebraic process systems AIChE J. 1987, 33, 1257-1270. [CrossRef]

31. Gopal, M . Control Systems: Principles and Design; Tata McGraw-Hill Education: New York, NY, USA, 2002.

32. Bracewell, R. The Fourier Transform and Its Applications; McGraw-Hill: Boston, MA, USA, 2000.

33. Melanie, M. An Introduction to Genetic Algorithms; MIT Press: Cambridge, MA, USA, 1996.

34. Mahmoud, E.E.; Al-Harthi, B.H. A hyperchaotic detuned laser model with an infinite number of equilibria existing on a plane and its modified complex phase synchronization with time lag. Chaos Solitons Fractals 2020, 130, 109442. [CrossRef]

35. Mahmoud, E.E.; AL-Harthi, B.H. A phenomenal form of complex synchronization and chaotic masking communication between two identical chaotic complex nonlinear structures with unknown parameters. Result. Phys. 2019, 14, 102452. [CrossRef]

36. Mahmoud, E.E.; Althagafi, O.A. A new memristive model with complex variables and its generalized complex synchronizations with time lag. Results Phys. 2019, 15, 102619. [CrossRef]

Publisher's Note: MDPI stays neutral with regard to jurisdictional claims in published maps and institutional affiliations.

(C) 2020 by the authors. Licensee MDPI, Basel, Switzerland. This article is an open access article distributed under the terms and conditions of the Creative Commons Attribution (CC BY) license (http:/ / creativecommons.org/licenses/by/4.0/). 